\title{
La injusticia cognitiva en la división internacional del conocimiento. El caso de la migración altamente cualificada
}

Cognitive Injustice in the International Division of Knowledge. The Case of Highly Skilled Migration

L'injustice cognitive dans le partage international du savoir. Le cas de la migration hautement qualifiée

\section{Francesco Maniglio}

\section{OpenEdition}

\section{Journals}

Edición electrónica

URL: http://journals.openedition.org/rccs/6747

DOI: $10.4000 /$ rccs. 6747

ISSN: 2182-7435

Editor

Centro de Estudos Sociais da Universidade de Coimbra

Edición impresa

Fecha de publicación: 1 diciembre 2017

Paginación: 27-46

ISSN: 0254-1106

Referencia electrónica

Francesco Maniglio, « La injusticia cognitiva en la división internacional del conocimiento. El caso de la migración altamente cualificada », Revista Crítica de Ciências Sociais [En línea], 114 | 2017, Puesto en línea el 20 diciembre 2017, consultado el 30 abril 2019. URL : http://journals.openedition.org/ rccs/6747; DOl : 10.4000/rccs.6747 


\section{FRANCESCO MANIGLIO}

\section{La injusticia cognitiva en la división internacional del conocimiento. El caso de la migración altamente cualificada}

El aumento de los flujos migratorios de personas altamente cualificadas, indica la consolidación mundial de una demanda de mano de obra migrante en los sectores de I+D, ICT, servicios financieros, ciencia y tecnología. En este proceso, para sustentar los argumentos de liberalización del mercado del trabajo global, las posiciones desarrollistas continúan siendo dominantes. Por ejemplo, según el Banco Mundial, la migración altamente cualificada y el capital humano incrementan la democracia y la libertad económica. El objetivo de la presente investigación es analizar, desde la perspectiva de la Economía Política, la gran relevancia asumida por las migraciones altamente cualificadas en el desarrollo global de la economía del conocimiento. Argumentamos en qué medida los mecanismos de inclusión diferencial determinan unos flujos de talentos direccionales y no circulares. La injusticia social global está, por lo tanto, íntimamente unida a la injusticia cognitiva global: la migración altamente cualificada se coloca así dentro del movimiento neocolonial del capitalismo del conocimiento.

Palabras clave: división internacional del conocimiento; economía del conocimiento; justicia cognitiva; migraciones; trabajadores cualificados.

Palavras-chave: divisão internacional do conhecimento; economia do conhecimento; justiça cognitiva; migrações; trabalhadores qualificados.

\section{Introducción}

Los procesos migratorios han ido ganando cada vez mayor importancia en términos económicos, políticos y sociales, hasta el punto en que existe un consenso generalizado para definir las ultimas dos décadas como la época de la migración (Castles, Haas y Miller, 2013). Se trata de un consenso entorno al hecho de que las migraciones devienen fundamentales para el crecimiento económico y para el desarrollo social en todo el mundo. Globalmente estamos siendo testigos del proceso exponencial de financiarización de la economía, con la deslocalización y el desmantelamiento gradual de los 
sistemas de seguridad social, con nuevas dependencias productivas y la movilidad de una fuerza de trabajo en continua formación (Sierra Caballero y Maniglio, 2016). La mayoría de la población migrante no viene de los países más pobres ni de las clases sociales más pobres. Son empresarios o especialistas altamente cualificados. La migración internacional deviene un activo de mercado siempre más estratégico, que requiere de políticas y recursos significativos. Los procesos de regulación de los flujos de capital-conocimiento permiten a los países de destino competir para atraer a los altamente cualificados a través de una normativa privilegiada para la entrada y la residencia. Los gobiernos, en efecto, siguen manteniendo el poder de la organización sistémica de la inteligencia colectiva tanto en la protección de los derechos de propiedad intelectual (para controlar o favorecer las monopolizaciones) como en la regulación de las agencias formativas (las universidades y los centros de investigación) y en la regulación de los flujos del capital humano. Un Estado que invierte sobre la empresarialidad y la empleabilidad de los estudiantes que devienen de esta forma sujetos activos del capitalismo académico (Mars, Slaughter y Rhoades, 2008). En este sentido, investigar las migraciones cualificadas significa investigar los mecanismos de valorización y reproducción del capital cognitivo: los mecanismos políticos (gobierno financiero y gobernanza cognitiva) y las nuevas características económico-sociales (mercado de trabajo-inclusión diferencial). Hablamos en los términos de un neocolonialismo del conocimiento -o en palabras de González Casanova, de un colonialismo inter, intra y transnacional- porque en esta transformación persisten las ideologías de la economía política clásica, al querer transformar los sistemas educativos en empresas financieras adeptas a la producción de escasez, en una situación en la que existe una abundancia y una riqueza potencial.

La colonización inter, intra y transnacional [...] permite aumentar su dominación mundial de los mercados y los trabajadores, así como controlar en su favor los procesos de distribución del excedente en el interior de cada país, en las relaciones de un país con otro y en los flujos de las grandes empresas transnacionales. La política globalizadora y neoliberal redefine las empresas y los países con sus redes internacionales, intranacionales y transnacionales. El mundo no puede ser analizado si se piensa que una categoría excluye a las otras. (González Casanova, 2006: 425)

En esta aportación presentamos una reflexión sobre los procesos neocoloniales representados por las migraciones cualificadas, las relaciones de estos procesos con las políticas económicas y con los intereses nacionales en los sectores de la educación superior, la investigación y el desarrollo, 
la innovación, la tecnología y los servicios financieros. La hipótesis de un neocolonialismo protagonizado por las migraciones cualificadas recusa las perspectivas generalizadoras de un brain gain, que presumen de los efectos positivos a largo plazo en caso de un posible retorno, o de una brain circulation que defiende que las redes de las elites emigradas (Hunger, 2002) producen un capital social que permite obtener ventajas regionales en una economía global (Saxenian, 2007). Nuestro enfoque responde a la necesidad de enfrentar la problemática de las migraciones cualificadas desde la materialidad del trabajo, y la valorización de esta fuerza-trabajo desde la perspectiva de la desigualdad y la injusticia social.

En primer lugar analizamos los flujos de migraciones cualificadas en relación con las determinantes políticas de la economía del conocimiento: (1) cómo las políticas tecnológicas y de innovación desarrollan un papel estratégico en determinar los flujos de entrada y salida de los trabajadores extranjeros cualificados; (2) cómo las políticas de las universidades y de los institutos de investigación -en la definición de las cuotas de inmigración- refuerzan la influencia global de determinados países en la producción y reproducción del dominio político-económico (los intereses político-corporativos).

En segundo lugar insistimos en cómo las políticas nacionales de ciencia y tecnología dan lugar a nuevas formas culturales y materiales de colonización y dominación. Desde la perspectiva de la economía política del conocimiento, consideramos los enfoques del Banco Mundial y de la OCSE como parte de un monocultivo institucional, al seguir valorando generalmente como positivo el impacto de la migración cualificada.

El monocultivo institucional descansa tanto en la premisa general de que la eficacia institucional no depende de los ajustes socioculturales locales, como en la premisa más específica de que las versiones idealizadas de las instituciones angloamericanas son los instrumentos óptimos para el desarrollo, independientemente del nivel de desarrollo o de la posición en la economía global. (Evans, 2004: 33$)^{1}$

En contraposición, indagamos la injusticia cognitiva (Santos, 2009) desde el punto de vista de la hemorragia o fuga de cerebros, porque se inscribe en una dinámica más amplia de injusticia social global. Esta se centra en la contradicción entre las políticas selectivas de inmigración y el discurso de la movilidad, la democracia y los derechos humanos que determinan las direcciones de los flujos de migraciones cualificadas, consolidando una específica división internacional del conocimiento.

${ }^{1}$ Traducción del autor. 


\section{La brain migration y los flujos internacionales de conocimientos}

El término "fuga de cerebros" fue acuñado en Gran Bretaña en los años sesenta, cuando pareció que el flujo se había invertido; por ejemplo, los científicos e ingenieros británicos emigraron a Estados Unidos, mientras que los indios llegaron al Reino Unido [...]. Acusados de "caza furtiva", los países beneficiarios -en primer lugar los Estados Unidos- argumentaron, con las armas teóricas de la teoría del capital humano, que los países de origen no estaban heridos. (Vinokur, 2006: 7)²

El interés político-económico y académico sobre las migraciones altamente cualificadas ha crecido exponencialmente en los últimos años. Este interés se debe a la dependencia de las economías "occidentales" respecto a los expertos y científicos extranjeros (Blitz, 2010: 3296-3320). Slaugher y Rhoades (2004), en Academic Capitalism and the New Economy: Markets, State and Higher Education, enfatizan esta dependencia analizando los procesos capitalistas en tanto que regímenes de conocimiento y aprendizaje; es decir, cómo los estudiantes, a través del aprendizaje formal e informal, se están transformado paulatinamente en empresarios de sí mismos. Numerosos estudios se han focalizado en el papel de los estudiantes y en los factores que influyen a la hora de elegir las instituciones de educación superior en términos de competencias, probabilidad selectiva y desarrollo cuantitativo entre instituciones (Kose, 2012). Los intentos de los Estados o universidades de atraer a estudiantes extranjeros ${ }^{3}$ se enmarcan entre motivaciones y objetivos que van desde lo económico (diferencial de renta) y lo cultural (en términos de captura cognitiva), a los mecanismos de reproducción de clase (en términos neocoloniales). Por esto queremos analizar el papel de la migración altamente cualificada dentro de los flujos migratorios generales, destacando la consolidación de unas estructuras reguladoras nacionales e internacionales.

A diferencia de los estudios marxista clásicos y de la teoría crítica de las migraciones (Piore, 1979; Castles y Kosack, 1972) que se basan en el modelo dual del mercado laboral, nosotros nos centramos en los procesos de división internacional del trabajo humano altamente cualificado: ${ }^{4}$ en cómo las regulaciones internacionales del conocimiento devienen las armas estratégicas de los gobiernos, en los conflictos en torno a las nuevas formas de valorización y explotación del capital.

\footnotetext{
2 Traducción del autor.

${ }^{3}$ Pensemos, por ejemplo, en los early decision programs, con los cuales las universidades intentan capturar a los mejores estudiantes, ofreciendo una seguridad de admisión con antelación; sobre todo con respecto a las ayudas financieras, elemento importantísimo para aquellas generaciones de estudiantes con bajo estatus social o provenientes de institutos de bajo prestigio.

${ }^{4}$ Cfr. Francesco Maniglio, 2016.
} 
No nos focalizamos más sobre la inequidad absoluta sino sobre las inequidades relativas, y cómo estas intervienen en cuanto mecanismos selectivos de migración. ${ }^{5}$ Desde la década de los noventa, el mercado de trabajo para cualificados se ha ido extendiendo en términos transfronterizos, aumentando progresivamente el número de cuotas en los países "occidentales" e implementando los programas de movilidad internacional en la mayoría de las universidades (Stein et al., 1996). En estos países, el aumento de la demanda de profesionales cualificados expandió el campo de reclutamiento hacia el exterior, generando un marco regulativo y competitivo internacional. Se habla en términos de una "guerra" para dirigir los flujos migratorios altamente cualificados, impulsada por países, empresas y universidades, a través de políticas migratorias para la atracción y la retención de personal cualificado (Boeri et al., 2012: 25-30). El fenómeno del brain drain proporciona las coordenadas visuales de esta guerra y de las divisiones a que nos estamos refiriendo.

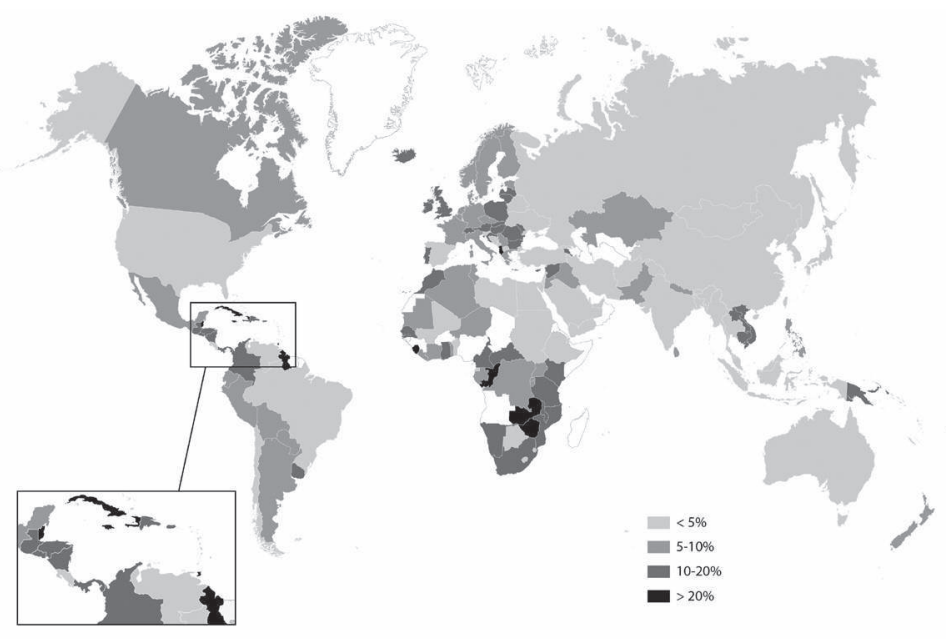

MAPA 1 - El fenómeno Brain Drain en el $2010^{6}$

Fuente: OECD, 2011.

El nivel de discriminación de los inmigrantes se valoriza en el mercado laboral y en las cuentas públicas de aquellos países que adoptan políticas

\footnotetext{
${ }_{5}$ Cfr. Yvonne Stolz y Joerg Baten, 2012.

${ }^{6}$ En orden cronológico es el último dato global que podemos visualizar en diciembre de 2016.
} 
selectivas de inmigración, estableciendo flujos migratorios controlados y reduciendo la inmigración no cualificada. La clasificación de "trabajadores prioritarios" ha sido adoptada por los países occidentales con el objetivo de discriminar a los deseados (personal altamente cualificado) con respecto a los no deseados (personal no cualificado). Hablamos de Canadá, Australia, Japón y la UE, cuyas políticas de inmigración -especialmente desde la década de los noventa- se han vuelto selectivas de acuerdo con las exigencias del mercado de trabajo interno. Pero, sobre todo, nos referimos a EE. UU., donde los estudiantes internacionales representan la cuota más alta respecto al número total de visados temporales expedidos:

TABLA 1 - Migración temporal en EE. UU. (en miles/ personas)

\begin{tabular}{|c|c|c|c|c|}
\hline & 2005 & 2013 & 2014 & $\begin{array}{c}\text { Media } \\
2009 / 2013\end{array}$ \\
\hline Estudiantes internacionales & 237,9 & 534,3 & 595,6 & 437,0 \\
\hline Trabajadores en formación & 1,8 & 2,7 & 22 & 2,3 \\
\hline Trabajadores en vacaciones & 88,6 & 86,4 & 90,3 & 99,7 \\
\hline Trabajadores estacionales & 31,9 & 74,2 & 89,3 & 62,2 \\
\hline Movilidad entre-empresarial & 65,5 & 66,7 & 71,5 & 67,9 \\
\hline Otros trabajadores internacionales & 266,1 & 275,7 & 296,8 & 237,0 \\
\hline
\end{tabular}

Fuente: OECD, 2016.

En el ámbito europeo, el comisario Frattini lanzó en el 2007 la iniciativa de la tarjeta azul europea (Directiva 2009/50/CE), que se corresponde -con las debidas diferencias- con la carta verde de EE. UU., para favorecer el ingreso de inmigrantes altamente cualificados; dando la razón, de esta forma, a las medidas ya adoptadas por Alemania, Reino Unido y Francia, que a su vez habían seguido los pasos de EE. UU., Canadá y Australia. ${ }^{7}$

\footnotetext{
${ }^{7}$ Australia y Canadá fueron los pioneros en adoptar estos tipos de medidas, implementando desde los años sesenta y setenta un sistema de visados con puntos basados en los skills. El sistema de puntos fue establecido en Australia en 1958 con The Migration Act, en base a criterios de edad, titulaciones, experiencia y salud. En 1978, Canadá adoptó el mismo modelo australiano con el Immigration Act. Estas políticas tuvieron como consecuencia que en 1997, más de la mitad de la inmigración en Canadá estuviese compuesta por profesionales altamente cualificados. El sistema de EE. UU. sigue las líneas de los anteriores, funcionando por cuotas de visados. Esta cuota, fijada mediante el Inmigration Act en 65 mil unidades, fue incrementada en la década de los noventa a 115 mil, durante la presidencia de Clinton, con el American Competitiveness Work Force Improvemet Act, en consonancia con el desarrollo de las políticas sobre la Sociedad de la Información.
} 


\section{Migraciones altamente cualificadas y déficit tecnológico}

Las políticas públicas de inmigración de personas altamente cualificadas devienen un segmento inseparable de las políticas de desarrollo tecnológico nacionales. La direccionalidad de la movilidad humana es consecuente con el proceso selectivo de la inmigración. Las políticas gubernamentales y las políticas de los grupos de presión financieros intervienen incrementando el poder de atracción de los países receptores a través de los incentivos fiscales, el aumento de las cuotas salariales, las infraestructuras y los núcleos de investigación. El Global Talent Index -elaborado por el Economist Intelligence Unit- subraya especialmente la geografía de inclusión diferencial del capital humano, indicando cómo:

[...] los EE. UU. y los países nórdicos son los más performativos, en los índices de 2011 y 2015. En su capacidad de producir y atraer el talento, los EE. UU. están muy por delante de otros países, casi un punto por delante de su competidor más cercano (en una escala con índice de 1-10). Está situado en la parte superior en los índices del 2011 y conserva su posición preeminente en el año 2015. La excelencia de sus universidades es un factor importante en esta actuación. Un tercio de las universidades clasificadas en la top 500 del mundo se encuentran en los EE. UU., que por lo tanto producen una serie consistente de graduados muy competitivos para prosperar en una economía del conocimiento. (Economist Intelligence Unit, 2011: 7)

Una geografía que vincula muy fuertemente los flujos internacionales de trabajadores altamente cualificados con los factores principales de desarrollo de la economía global y con la competitividad de las economías nacionales. Las consecuencias de estas relaciones se reflejan en la direccionalidad de los flujos migratorios, particularmente en las consecuencias de lo que se denomina como brain drain y brain wastes (Koser y Salt, 1997: 298-299). La capacidad de algunos países, regiones y ciudades para atraer personal de talento es enorme, como es enorme la dependencia a la que muchos gobiernos están condenados para reaccionar frente a estos escenarios. Se hace cada vez más tajante la desigualdad entre las naciones para retener, atraer y suplir los flujos de personas altamente cualificadas. Específicamente y considerando el mapa mundial sobre la brecha entre el crecimiento de la demanda y de la oferta, se pueden distinguir varias consideraciones políticas; entre estas, las teorías neoclásicas de la convergencia económica y del technological gap approach (Fagerberg, 1987; Romer, 1993) consideran el balance de talentos en términos de déficit de convergencia en el desarrollo de una economía del conocimiento. Los flujos de migrantes altamente cualificados se podrían interpretar -según estos estudios- como unidireccionales entre un norte y un sur global siempre más dependientes. 


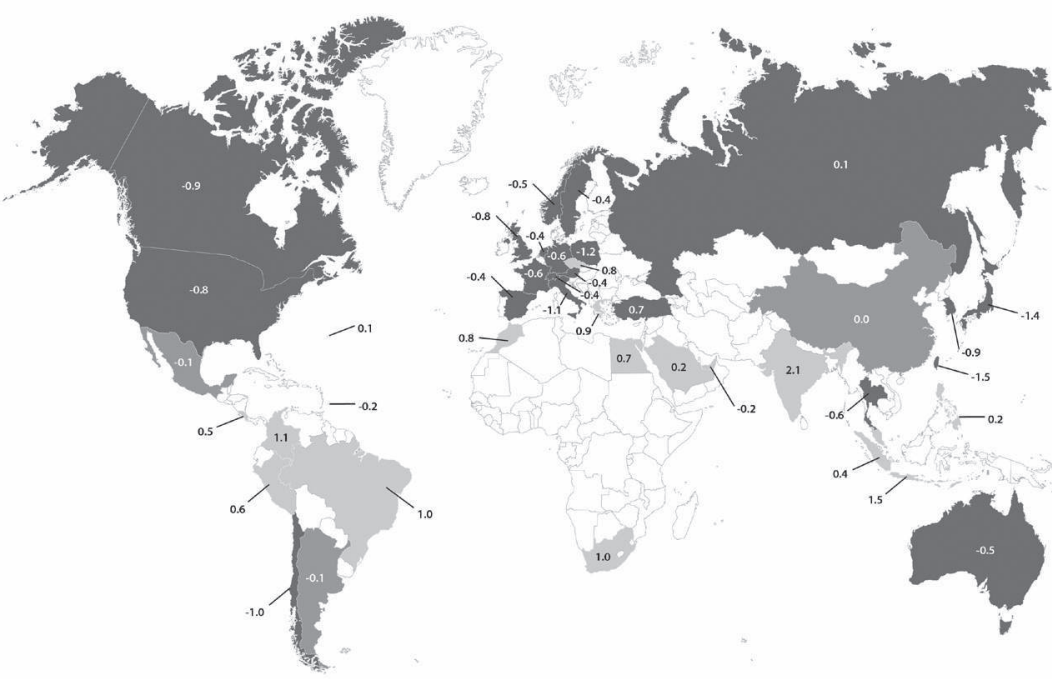

MAPA 2 - La brecha entre el crecimiento de la demanda y el crecimiento de la oferta de talento, 2011-2021

Fuente: Oxford Economics, 2012.

Nota: En negro indicamos la tendencia de déficit, en gris oscuro la tendencia de superávit y en gris claro el equilibrio. Los números muestran la tendencia de crecimiento en porcentajes anuales.

Efectivamente, en las políticas tecnológicas de los países "occidentales", se considera siempre más importante y estrecha la relación entre transferencia de tecnologías, movilidad y cooperación social, en términos de capital humano. Sin embargo, no se trata de un fenómeno altruista y filantrópico, como se nos presenta con el concepto de una diáspora científica que beneficia a los países "en vía de desarrollo" (Barre et al., 2003; Meyer, 2001), o que incluso determina un cambio hacia un modelo de "desarrollo sustentable” (Tejada y Bolay, 2010). Los Estados luchan con el objetivo de no perder la ventaja comparativa en una competición global de la innovación, la ciencia y la tecnología. Los sistemas nacionales de innovación de estos países, representan parte de los dispositivos de la lucha a la que nos estamos refiriendo, al distinguir las cuotas de los trabajadores extranjeros cualificados. En el periodo 1996-2006, por ejemplo, los EE. UU. han retenido ingenieros y profesionales en el campo de la ciencia y tecnología con una tasa tres veces mayor que en otras ocupaciones. En este proceso selectivo son las universidades las que 
detentan el papel protagonista, en tanto que cerca del $70 \%$ de los estudiantes que terminan la formación de doctorado (ciencias e ingenierías) permanece por estadías medio-largas, y la mayoría se quedan permanentemente (Finn, 2014).

\section{Universidad, movilidad y retención de talentos}

Históricamente las universidades y los institutos de investigación detentan un papel protagónico en la definición de las cuotas migratorias de cada país, porque intervienen directamente en las disputas para la captación y explotación del capital humano. La "lucha por los talentos", analizada por la McKinsey Quarterly, se constata en el aumento del porcentaje de estudiantes de educación terciaria que se matricularon fuera de su país de ciudadanía, que ha pasado del $2 \%$ en 1950 al 2,3\% en 1970, alcanzando el 3,8 \% en 1990 (OECD, 2012). En el 2016, el 6 \% de los estudiantes matriculados en la educación terciaria de los países de la OCDE eran estudiantes internacionales.

Mahroum (2005) los define como pasajeros de clase económica, porque se encuentran empujados sobre todo por factores económicos, o sea, por las mejores ofertas en términos comparativos. En términos geopolíticos, los países que atraen más estudiantes de educación superior son aquellos que más retienen a los trabajadores altamente cualificados. En el 2016, el número de estudiantes extranjeros matriculados en educación terciaria en los países miembros de la OCDE era, como media, tres veces superior al de los estudiantes de los países de la OCDE que estudiaban en el extranjero. Los asiáticos representan el $53 \%$ de los estudiantes extranjeros matriculados en todo el mundo ${ }^{8}$ (OECD, 2016).

Por lo tanto, en la distribución de los estudiantes extranjeros de educación superior, podemos observar algunos aspectos de la división internacional del conocimiento: en particular, Estados Unidos captura el mayor número de estudiantes internacionales a nivel de maestría y doctorado ( $26 \%$ del total), seguido por el Reino Unido (15\%), Francia (10\%), Alemania (10\%) y Australia $8 \%$ (OECD, 2016: 328). Estas tendencias describen el consenso en torno a la disputa por la captación de capital humano altamente cualificado. ${ }^{9}$ Sin embargo, consenso no significa convergencia. Regresemos un momento al mapa número 2 , sobre la brecha entre el crecimiento de la demanda y el crecimiento de la oferta de talento. Observamos cómo EE. UU., Australia y la Unión Europea comparten valores similares -EE. UU. un 0,8 , Australia y la UE un $0,5-$ pero

\footnotetext{
${ }^{8}$ Los estudiantes de China representan el $22 \%$ de todos los estudiantes internacionales matriculados en los niveles de maestría y doctorado, o equivalentes, en el área de la OCDE. El 41 \% se encuentra en Estados Unidos, mientras que el 39 \% se reparte entre Australia, Francia, Alemania y el Reino Unido.

9 Este consenso se construye con la intersectorialidad de las políticas en materia de seguridad e inmigración, las políticas industriales, de educación, de innovación y desarrollo, y las políticas del mercado de trabajo de los gobiernos con más demanda.
} 
que no describen una convergencia en el desarrollo de una economía del conocimiento. Todo lo contrario, indican unas estrategias de escala múltiple que compiten en los sectores de la educación superior, la ciencia y las tecnologías.

Si tomamos el caso de Australia, por ejemplo, desde los años noventa el objetivo estratégico de las políticas educativas fue el crecimiento del volumen y la expansión de las matrículas extranjeras. Desarrollar el carácter económico de la educación como industria específica del conocimiento, estableciendo acuerdos internacionales con los países de procedencia y creando una nueva política de inmigración, representa el éxito de una forma particular de valorización y explotación del conocimiento. Pensemos, por ejemplo, que "entre 1990 y el 2003, la participación de Australia en el mercado mundial de títulos creció del 1 al 9 \%" (Marginson, 2007), y en el 2014, el 18 \% de los estudiantes universitarios eran internacionales (OECD, 2016).

\section{TABLA 2 - Movilidad internacional de estudiantes y estudiantes extranjeros en educación terciaria (2014)}

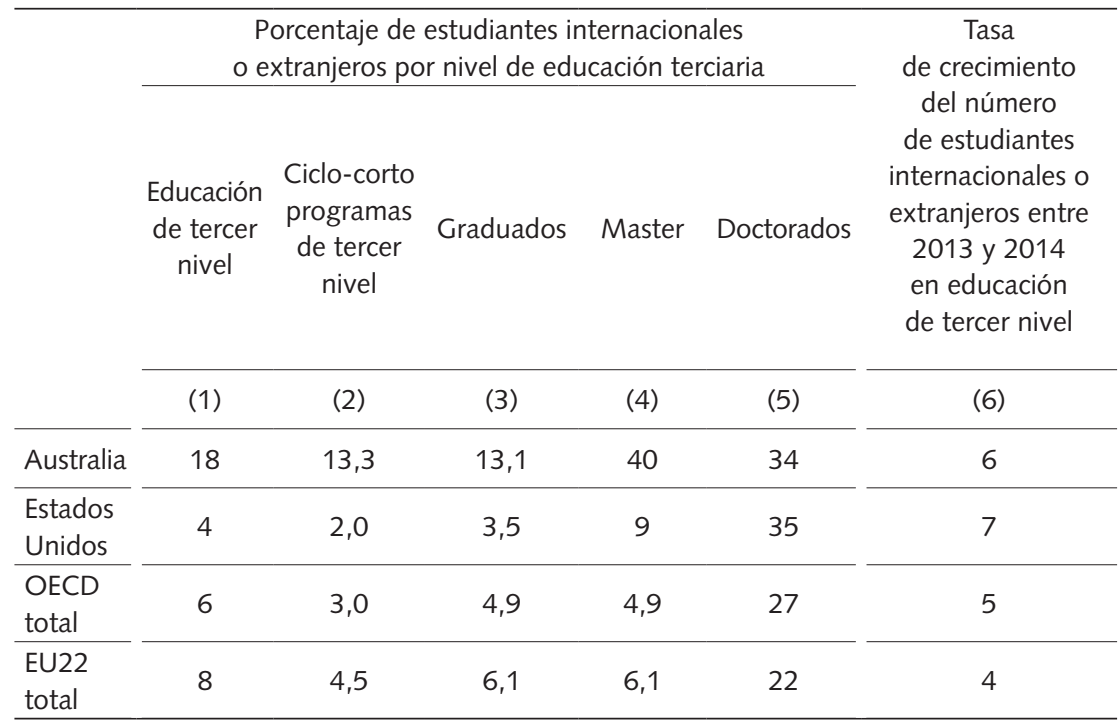

Fuente: OECD, 2016

Mientras tanto, las políticas de educación superior e investigación de EE. UU. se concentran en abordar el mercado global de la educación doctoral y posdoctoral. En el 2014, el 35 \% de los estudiantes de doctorado eran extranjeros. En definitiva, Estados Unidos tiene más estudiantes de doctorado extranjeros que el resto del mundo junto. Este liderazgo 
estadounidense en el mercado de la educación doctoral, puede explicarse por los flujos de capital que financian universidades y centros de investigación, tanto en el país como en el extranjero.

Las universidades participan en la creación de nuevas empresas, que supuestamente ayudan a optimizar los beneficios mediante la transferencia de tecnología y conocimiento. ${ }^{10}$

Ya en el año 2000, más del $50 \%$ de los posdoctorados en ciencias e ingeniería que se especializaban en estas instituciones provenían del extranjero; y en Silicon Valley, el $30 \%$ de los profesionales informáticos han nacido en el exterior (Mahroum, 2000). Sin embargo, los estudiantes europeos que se han doctorado en EE. UU. tienen una tasa de permanencia mucho más alta con relación a los de Corea o Japón. Esta división hacia los centros y las élites del conocimiento ilustra cómo cerca del $60 \%$ de los doctorados de EE. UU. de origen europeo se quedan ahí, respecto a los doctorados coreanos y japoneses, ya que sólo permanece un tercio del total de estos últimos. China e India son los Estados más afectados por esta pérdida, dado que el $85 \%$ y el $82 \%$, respectivamente, de los doctorados de estas nacionalidades se quedan en los EE. UU. (National Science Board, 2014).

TABLA 3 - Porcentaje de residentes temporales (europeos) que recibieron doctorados de Ciencias e Ingeniería en el 2006 y que han residido en los Estados Unidos entre el 2007 y el 2011, por país de origen

\begin{tabular}{lccccc}
\hline País & $\mathbf{2 0 0 7}$ & $\mathbf{2 0 0 8}$ & $\mathbf{2 0 0 9}$ & $\mathbf{2 0 1 0}$ & $\mathbf{2 0 1 1}$ \\
\hline Grecia & 60 & 55 & 53 & 53 & 47 \\
\hline Reino Unido & 74 & 67 & 69 & 66 & 64 \\
\hline Alemania & 67 & 66 & 61 & 56 & 53 \\
\hline Italia & 64 & 61 & 59 & 59 & 57 \\
\hline Francia & 64 & 62 & 62 & 56 & 62 \\
\hline Rumania & 90 & 88 & 87 & 84 & 83 \\
\hline España & 55 & 52 & 44 & 47 & 47 \\
\hline Otros países de UE & 55 & 50 & 50 & 50 & 48 \\
\hline
\end{tabular}

Fuente: Finn, 2014.

\footnotetext{
${ }^{10}$ Los proof-of-concept centers, como la Universidad de California, San Diego, el Centro William von Liebig, han conseguido incubar una serie de startups desde 2001. Las incubadoras de empresas se han multiplicado, desde la Universidad de Utah hasta la Universidad de Kansas y de South California. La presencia en la bahía de San Francisco de dos grandes centros de innovación, como Stanford y Berkeley, es crucial para entender el auge de Silicon Valley.
} 
Por esto analizamos los flujos migratorios internacionales de personas altamente cualificadas, según la jerarquía y las relaciones de poder de los países, "para la competitividad nacional y regional en la economía global, [porque] ha comenzado una batalla global por los talentos y los mercados que los sustentan" (Robertson y Keeling, 2008: 14).

En el caso de la UE, con las políticas de selectividad de los inmigrantes masivos, se han impuesto también una serie de medidas para el reconocimiento más rápido de las titulaciones académicas, para facilitar la competitividad entre los centros de educación superior. Se crea el Espacio Europeo de Educación Superior (EEES), según ventajas comparativas respecto al modelo de los EE. UU., a través de reformas progresivas trazadas desde la Declaración de Bolonia. ${ }^{11}$ El objetivo es ampliar los mecanismos de movilidad con las políticas selectivas para la migración cualificada y atraer a "los mejores profesores e investigadores, de contratarlos a través de procedimientos flexibles, abiertos y transparentes, de garantizar a los investigadores principales y los jefes de equipo una independencia total para investigar, y de ofrecer a su personal unas perspectivas de desarrollo profesional atractivas" (Comisión de las Comunidades Europeas, 2006: 10).

El proyecto europeo de educación superior, percibido cada vez más como significativo para la economía mundial, ha desencadenado una serie de reacciones dinámicas en países como Australia y los Estados Unidos, lo que conduce a múltiples lógicas y nuevos imaginarios sobre la globalización de la educación superior. A través de este [proyecto] está emergiendo un sistema global más integrado de educación superior (Robertson y Keeling, 2008: 3). ${ }^{12}$

Una estrategia global que al mismo tiempo promueve e intensifica los mecanismos de competición y dependencia intrarregional. Una dependencia que arroja el riesgo concreto de una monocultura en la UE (Bronk y Jacoby, 2013) y no sólo en lo que concierne la relevancia de la unión monetaria y fiscal (consenso alemán). Hablamos de la direccionalidad de los flujos de doctorados a nivel intraeuropeo desde las regiones del sur -que pierden continuamente su capital-conocimiento- hacia los países centrales europeos (y en el ámbito

\footnotetext{
${ }^{11}$ (1) planes de estudio a escala europea que permitan la globalización de las competencias curriculares básicas; (2) el desclasamiento continuo del conocimiento, a través de la multiplicación de grados y diplomas; (3) currículos más largos por duración, fomentando nuevos flujos de financiamiento a la empresa-universidad, la formación de una deuda pedagógica y la precarización de estudiantes/investigadores; (4) la institución de un sistema de educación superior de tres ciclos (ISCED 5-6-7-8) (Maniglio, 2016).

12 Traducción del autor.
} 
extraeuropeo, hacia los EE. UU.). En una reciente investigación (Wende, 2015) sobre la concentración de talentos en Europa se hizo énfasis en el caso de Grecia, que pierde capital humano (el $73 \%$ de los migrantes tiene un título universitario y el $51 \%$ un doctorado) en favor del Reino Unido (31 \%), EE. UU. (28\%) y Alemania. Italia también pierde muchos cualificados, principalmente a favor de EE. UU. (34 \%), Reino Unido (26\%) y Francia (11 $\%)$. La investigación detecta como principales las razones estructurales, tales como "la falta de financiación para la investigación respecto a mejores condiciones económicas y oportunidades de carrera en el extranjero" (ibidem: 79). En este contexto resulta difícil hablar de "circulación de cerebros" en tanto que fenómeno generalmente positivo, cuando son las estructuras y las redes profesionales (capital social acumulado) las que determinan las posibilidades concretas de regreso en los países de origen (Blitz, 2005: 377).

\section{Conclusiones: inclusión diferencial e injusticia social}

Decía Marx, en Das nacionales System der politischen Ökonomie, que "la nacionalidad del obrero no es francesa, ni inglesa, ni alemana en tanto que el trabajo es la esclavitud en libertad, es la venta voluntaria de sí mismo" (Marx y Engels, 1972: 585-614). Nosotros, al contrario que Marx, con esta aportación queremos afirmar y subrayar cómo las cuestiones -y divisionesrelativas a la nacionalidad devienen estratégicas para reforzar la competencia global entre los Estados, para la valorización del capital-conocimiento y, sobre todo, para la generación de nuevas desigualdades e injusticias sociales. Hemos tenido la posibilidad de observar cómo una serie de regulaciones políticas y económicas (permisos permanentes como la green card o la tarjeta azul, contexto formativo atractivo, contexto laboral económicamente estimulante) han consolidado una particular división internacional del conocimiento y han puesto en un lugar central la cuestión económico-política del regreso a los países de origen. Las políticas públicas de los Estados "occidentales" sobre ciencia y tecnología, seguridad e inmigración asumen una relevancia estratégica para la economía del conocimiento, amplificando los mecanismos de inclusión diferencial. En el caso de la crisis social de la Unión Europea, hablamos de un dilema político (Schierup, Hansen y Castles, 2006) consecuencia del declino del estado de bienestar, que ya es incapaz de mantener una idea de equidad social, y de los Estados nacionales incapaces a su vez de gestionar el incremento de las desigualdades. En este cuadro regulatorio, hemos visto como la hipótesis más equitativa de una "circulación de cerebros" es difícil de corroborar si consideramos que en muchos países de origen se registra, en efecto, una "hemorragia de inteligencias", un "drenaje de cerebros": 
Encontramos que el crecimiento de la migración contribuye a mejorar la calidad institucional (medida por indicadores estándar de democracia y libertad económica) en los países de origen de los migrantes. Este resultado es bastante fuerte gracias a la exclusión de ciertos grupos de países (por ejemplo, los países del antiguo bloque socialista). (Docquier et al., 2011: 33)13

Históricamente los flujos migratorios han consolidado la geografía de la dependencia entre países que se denominaron desarrollados y subdesarrollados (Peña López, 1995). Una dependencia que sigue creciendo y produciendo otras estructuras de desigualdad como la gobernanza corporativa (Bebchuck y Roe, 2014) y la gobernanza cognitiva (Maniglio, 2016). El planteamiento de un mundo ideal donde viven los ciudadanos del conocimiento, circulando sin constricciones, se puede explicar sólo como un eslogan del Banco Mundial (World Bank, 2005a; 2005b: 205-224) para sustentar los argumentos de liberalización del mercado del trabajo global. Concretamente, hay que traer a la actualidad la teoría de la dependencia, porque los flujos de talentos son, efectivamente, multidireccionales pero en absoluto circulares (Beigel, 2013,2016; Cusicanqui et al., 2016). Si tomamos como casos emblemáticos los sistemas de salud de África y Centroamérica, ambos están afectados por el éxodo de médicos, enfermeros y técnicos que emigran hacia lugares más prometedores. Los resultados del estudio de Clemens (2007) sobre el Health Effects of African Health Professional Emigration, por ejemplo, denuncian cómo la emigración degrada los niveles de salud, incluso si los emigrantes son reemplazados por los nuevos profesionales. Es necesario admitir que dicha circulación ha permitido que las corporaciones multinacionales puedan disponer de un amplio contingente de trabajadores científicos y tecnológicos provenientes de lo que se denomina como Sur global, capitalizando altos beneficios mediante el desarrollo de nuevas tecnologías y la concentración de patentes (Martínez Pizarro, 2008; OIM, 2016). Sin embargo, en la mayoría de estos casos, las empresas están relacionadas o son extensiones de multinacionales (la mayoría de los polos tecnológicos de EE. UU. y la UE) que explotan los talentos abaratando el coste de la fuerza de trabajo cualificada. La dependencia de la mayoría de los Estados de este Sur global se resume en la desesperación por atraer inversiones extranjeras, empresas globales y, actualmente, universidades globales. Estamos canalizando los escasos presupuestos educativos públicos en programas diseñados bajo los estándares de la sociedad del conocimiento, a costa de fortalecer nuevas dependencias cognitivas y desigualdades sociales. Considerando la perspectiva de la economía política del

13 Traducción del autor. 
conocimiento, debemos denunciar y luchar contra los enfoques teóricos como el brain gain o el brain circulation, y preguntarnos: ¿se puede continuar hablando de justicia global y de justicia cognitiva?

La injusticia social global está, por lo tanto, íntimamente unida a la injusticia cognitiva global. La batalla por la justicia social global debe, por lo tanto, ser también una batalla por la justicia cognitiva global. (Santos, 2009: 40)

Los izquierdistas progresistas europeos, los mismos que, al amparo de los Derechos Humanos, quieren crear una Europa atractiva para estudiantes de países en desarrollo bajo el ideal de la movilidad, del brain gain y del brain circulation, puntualmente se olvidan de los mecanismos de coerción que esas políticas públicas conllevan. Como bien denuncian Gaillard y Gaillard (2002) la migración internacional altamente cualificada es un fenómeno estrictamente político y la competitividad para los migrantes cualificados conlleva la creación de mecanismos regulatorios de selección incluyentes y desiguales. Los flujos de migraciones cualificadas refuerzan los procesos de inclusión diferencial de los individuos ${ }^{14}$ en el mercado del trabajo y del conocimiento global.

"No hay alternativa" afirmaba Franco Frattini, el mismo comisario europeo encargado de Justicia, Libertad y Seguridad que hace cuatro años, ante el Parlamento Europeo, promovía la otra cara del programa de la tarjeta azul: la vigilancia permanente de todas las fronteras exteriores para detectar a los inmigrantes y la instauración de "fronteras inteligentes", con el reconocimiento biométrico de todas las personas que entran y salen de Europa. Se denotan así las prácticas aporéticas sustentadas por el marco de los Derechos Humanos (DD. HH.); unas contradicciones angustiosas que ven, por ejemplo, cómo el Tribunal Europeo de Derechos Humanos $(\mathrm{TEDH})$ estipula claramente que la transferencia de inmigrantes a los centros de detención infringe sus derechos fundamentales, mientras que Frontex, una agencia ejecutiva de la UE, los envía directamente allí.

Este panorama se explica por un sinnúmero de razones estructurales. Pero todas ellas resultan difícilmente escindibles de unas políticas europeas y estatales basadas en la asfixia de la entrada legal, en el menosprecio de las solicitudes de asilo, en el control de las zonas de tránsito por medio de sistemas militarizados, en la construcción de vallas y muros cada vez más altos y sofisticados, en las devoluciones masivas, o en crecientes retornos en frontera. (Aparicio Wilhelmi y Pisarello Prados, 2006: 111)

${ }_{14}$ Para profundizar las cuestiones concernientes a la inclusión diferencial en el mercado laboral cfr. Francesco Maniglio, 2016. 
El objetivo de esa ciudadanía es de crear una fortaleza manteniendo el cierre social y político a un buen número de sujetos. Se alimenta esta postura discriminante afirmando que "la política de la ciudadanía para todos -sin mirar a quién - no solo es una política destinada al fracaso, sino que además es una política que agrava y convierte en explosivos los problemas que se pretende resolver" (Sartori, 2001: 118). Se propone una ciudadanía exclusiva, con una selección de los inmigrantes según se les considere o no como integrables a determinadas funciones. En fin, el control de las fronteras se convierte en una máquina omnipresente, imperceptible, que divide constantemente a las personas entre deseables e indeseables, escribe Huub Dijstelbloem, en The Migration Machine (Dijstelbloem, Meijer y Besters, 2010). La injusticia social global se establece y se reproduce con este cuadro legislativo, donde los DD. HH. se limitan a los derechos del ciudadano, con la vulneración de "las más elementales exigencias del Estado de Derecho y de legitimidad democrática, domesticando -como advierte Habermas- el poder a través del derecho legitimado" (Solanes Corella, 2002: 118).

Revisado por José Morales

\section{Referencias bibliográficas}

Aparicio Wilhelmi, Marco; Pisarello Prados, Gerardo (2006), "Multiplicar las fronteras, externalizar el control”, Viento sur: por una izquierda alternativa, 89, 111-116.

Barre, Remi; Hernandez, Valeria; Meyer, Jean; Vinck, Dominique (2003), Scientific Diasporas: How Can Developing Countries Benefit from their Expatriate Scientists and Engineers. Paris: Institut de Recherche pour le Développement.

Bebchuck, Lucian; Roe, Mark (2014), "Una teoría sobre dependencia de caminos en propiedad y gobierno corporativo”, THĒMIS-Revista de Derecho, 46, 47-75.

Beigel, Fernanda (2013), "Centros y periferias en la circulación internacional del conocimiento”, Nueva Sociedad, 245, 110-123.

Beigel, Fernanda (2016), "El nuevo carácter de la dependencia intelectual", Cuestiones de sociología, 14.

Blitz, Brad (2005), "Brain Circulation: The Spanish Medical Profession and International Medical Recruitment in the United Kingdom”, Journal of European Social Policy, 15(4), 363-379.

Blitz, Brad (2010), "Highly Skilled Migration", in Robert A. Denemark (comp.), International Studies Encyclopedia. Oxford: Wiley-Blackwell, 3292-3320.

Boeri, Tito; Brücker, Herbert; Docquier, Frédéric; Rapoport, Hillel (2012), Brain Drain and Brain Gain: The Global Competition to Attract High-Skilled Migrants. Oxford: Oxford University Press. 
Bronk, Richard; Jacoby, Wade (2013), “Avoiding Monocultures in the European Union: The Case for the Mutual Recognition of Difference in Conditions of Uncertainty", LSE 'Europe in Question' Discussion Paper Series, 67.

Castles, Stephen; Haas, Hein de; Miller, Mark (2013), The Age of Migration: International Population Movements in the Modern World. Basingstoke: Palgrave Macmillan.

Castles, Stephen; Kosack, Godula (1972), "The Function of Labour Immigration in Western European Capitalism”, New Left Review, 73, 3-21.

Clemens, Michael (2007), "Do Visas Kill?: Health Effects of African Health Professional Emigration”, Center for Global Development, Working Paper n. ${ }^{\circ} 114$, March.

Comisión de las Comunidades Europeas (2006), Communication of 10 May 2006 from the Commission to the Council and the European Parliament - Delivering on the Modernisation Agenda for Universities: Education, Research and Innovation. Oficina de Publicaciones Oficiales de las Comunidades Europeas.

Cusicanqui, Silvia; Domingues, José Mauricio; Escobar, Arturo; Leff, Enrique (2016), "Debate sobre el colonialismo intelectual y los dilemas de la teoría social latinoamericana”, Cuestiones de sociología, 14.

Dijstelbloem, Huub; Meijer, Albert; Besters, Michel (2010), “The Migration Machine”, in Huub Dijstelbloem; Albert Meijer (comps.), Migration and the New Technological Borders of Europe. Basingstoke, Regno Unito: Palgrave, 1-21.

Docquier, Frédéric; Lodigiani, Elisabetta; Rapoport, Hillel; Schiff, Maurice (2011), Emigration and Democracy, Working Papers, Bar-Ilan University, Department of Economics, No. 2011-02.

Economist Intelligence Unit (2011), "The Global Talent Index Report: The Outlook to 2015”. S. 1.: Heidrick \& Struggles.

Evans, Peter (2004), "Development as Institutional Change: The Pitfalls of Monocropping and the Potentials of Deliberation", Studies in Comparative International Development, 38(4), 30-52.

Fagerberg, Jan (1987), “A Technology Gap Approach to Why Growth Rates Differ”, Research Policy, 16(2), 87-99.

Finn, Michael (2014), Stay Rates of Foreign Doctorate Recipients from US Universities, 2011. Oak Ridge, TN: Oak Ridge Institute for Science and Education.

Gaillard, Anne-Marie; Gaillard, Jacques (2002), "Fuite des cerveaux, circulation des compétences et développement: un enjeu politique”, Mots Pluriels, 20.

González Casanova, Pablo (2006), "Colonialismo interno (una redefinición)”, in Atilio A. Boron; Javier Amadeo; Sabrina González (comps.), La teoría marxista hoy: Problemas y perspectivas. Buenos Aires: CLACSO, 409-434.

Hunger, Uwe (2002), “The 'Brain Gain’ Hypothesis: Third World Elites in Industrialized Countries and Socioeconomic Development in their Home Country”, Center for Comparative Immigration Studies, Working paper 47. 
Kose, Ibrahim Alper (2012), “Student Loyalty in Higher Education Institutions”, Journal of Higher Education and Science, 2(2), 114-118.

Koser, Khalid; Salt, John (1997), "The Geography of Highly Skilled International Migration”, International Journal of Population Geography, 3(4), 285-303.

Mahroum, Sami (2000), "Highly Skilled Globetrotters: Mapping the International Migration of Human Capital", R\&D Management, 30(1), 23-32.

Mahroum, Sami (2005), "The International Policies of Brain Gain: A Review", Technology Analysis \& Strategic Management, 17(2), 219-230.

Maniglio, Francesco (2016), "Trabajo y plustrabajo en la sociedad del conocimiento. Algunas evidencias desde los procesos de financiarización de la economía”, in Francisco Sierra Caballero; Francesco Maniglio (comps.), Capitalismo financiero y comunicación. Quito, Ecuador: Ediciones Ciespal, 51-79.

Marginson, Simon (2007), "Global Position and Position Taking: The Case of Australia", Journal of Studies in International Education, 11(1), 5-32.

Mars, Matthew; Slaughter, Sheila; Rhoades, Gary (2008), "The State-sponsored Student Entrepreneur", The Journal of Higher Education, 79(6), 638-670.

Martínez Pizarro, Jorge (2008), América Latina y el Caribe: migración internacional, derechos bumanos y desarrollo. Santiago de Chile: CEPAL.

Marx, Karl; Engels, Friedrich (1972), Opere complete (Vol. 38). Roma: Editori Riuniti. Meyer, Jean Baptiste (2001), "Network Approach versus Brain Drain: Lessons from the Diaspora”, International Migration, 39(5), 91-110.

National Science Board (2014), Science and Engineering Indicators 2014 (No. NSB 14-01). Arlington, VA: National Science Foundation.

OECD (2011), DIOC 2010/2011: Database on Immigrants in OECD Countries. OECD Stat.

OECD (2012), Education at a Glance 2012: OECD Indicators. OECD Publishing.

OECD (2016), Education at a Glance 2014: OECD Indicators. OECD Publishing.

OIM - Organización Internacional para las Migraciones (2016), Estudio sobre migración calificada y desarrollo: Desafíos para América del Sur. Oficina regional para America el Sur.

Oxford Economics (2012), Global Talent 2021: How the New Geography of Talent Will Transform Human Resource Strategies. Oxford: Oxford University Press.

Peña López, Ana Alicia (1995), La migración internacional de la fuerza del trabajo (19501990): una descripción crítica. México: Editorial Cambio XXI.

Piore, Michael (1979), Birds of Passage: Migrant Labor in Industrial Societies. New York: Cambridge University Press.

Robertson, Susan; Keeling, Ruth (2008), Stirring the Lions: Strategy and Tactics in Global Higher Education. Bristol: Centre for Globalisation, Education and Societies, University of Bristol.

Romer, Paul (1993), “Idea Gaps and Object Gaps in Economic Development”, Journal of Monetary Economics, 32(3), 543-573. 
Santos, Boaventura de Sousa (2009), "Más allá del pensamiento abismal: de las lineas globales a una ecologia de saberes”, in VV. AA., Pluralismo epistemológico. La Paz: CLACSO-Muela del Diablo Editores, 31-84.

Sartori, Giovanni (2001), La sociedad multiétnica: Pluralismo, multiculturalismo y extranjeros. Mexico, DF: Taurus.

Saxenian, AnnaLee (2007), The New Argonauts: Regional Advantage in a Global Economy. London: Harvard University Press.

Schierup, Carl Ulrik; Hansen, Peo; Castles, Stephen (2006), Migration, Citizenship, and the European Welfare State: A European Dilemma. Oxford: Oxford University Press.

Sierra Caballero, Francisco; Maniglio, Francesco (coords.) (2016), Capitalismo financiero y comunicación. Quito, Ecuador: Ediciones Ciespal.

Slaughter, Sheila; Rhoades, Gary (2004), Academic Capitalism and the New Economy: Markets, State, and Higher Education. Baltimora: JHU Press.

Solanes Corella, Ángeles (2002), "Inmigración y derechos humanos”, Mediterráneo económico, 1, 105-120.

Stein, Anne Josephine et al. (1996), "International Education and Training of Scientists and Engineers and their Employment in European Industry". Bruxelles: PREST Report (European Commission).

Stolz, Yvonne; Baten, Joerg (2012), "Brain Drain in the Age of Mass Migration: Does Relative Inequality Explain Migrant Selectivity?”, Explorations in Economic History, 49(2), 205-220.

Tejada, Gabriela; Bolay, Jean Claude (2010), Scientific Diasporas as Development Partners: Skilled Migrants from Colombia, India and South Africa in Switzerland: Empirical Evidence and Policy Responses. Bern: Peter Lang.

Vinokur, Annie (2006), "Brain Migration Revisited", Globalisation, Societies and Education, 4(1), 7-24.

Wende, Marijk (2015), "International Academic Mobility: Towards a Concentration of the Minds in Europe”, European Review, 23(S1), S70-S88.

World Bank (2005a), Brain Gain: Claims about its Size and Impact on Welfare and Growth Are Greatly Exaggerated. Washington: World Bank, Development Research Group, Trade Team.

World Bank (2005b), World Development Report 2006: Equity and Development. Washington: World Bank Publications and Oxford University Press. 
Recibido: 08.03.2016

Aceptación comunicada: 05.09.2017

\section{Francesco Maniglio}

Centro Internacional de Estudios Superiores en Comunicación para América Latina

Av. Diego de Almagro N32-133 y Andrade Marín - Quito, Ecuador

Contacto: fmaniglio@ciespal.org

\section{Cognitive Injustice in the} International Division of Knowledge. The Case of Highly Skilled Migration

The increasing flux of international migration of highly qualified people points to the consolidation of the demand for a migrant labour force in the sectors of science and technology, R\&D, IT, and financial services worldwide. In this process, to support the arguments of liberalization of the global labour market, developmental positions always come to the fore. For example, according to the World Bank, highly skilled migration and human capital both serve to expand democracy and increase economic freedom. The objective of this research is to analyse, from the perspective of Political Economy, the very significant role played by the migration of the highly qualified in the global development of the knowledge economy. We argue how the mechanisms of differential inclusion determine flows of talent that are unidirectional and never circular. Global social injustice is closely linked to global cognitive injustice: the migration of highly qualified persons is thus well placed within the neo-colonial movement of the capitalism of knowledge. Keywords: cognitive justice; economy of knowledge; international division of knowledge; migrations; qualified workers.

\section{L'injustice cognitive dans le partage international du savoir. Le cas de la migration hautement qualifiée}

L'accroissement des flux migratoires de personnes hautement qualifiées marque la consolidation mondiale d'une demande de main-d'œuvre de migrants dans les secteurs d' I+D, ICT, des services financiers, de la science et de la technologie. Dans ce cadre, pour soutenir les arguments de libéralisation du marché du travail global, les positions développementalistes restent dominantes. Par exemple, selon la Banque mondiale, la migration hautement qualifiée et le capital humain accentuent la démocratie et la liberté économique. Le but de la présente recherche est d'analyser, du point de vue de l'Économie Politique, la grande importance que jouent les migrations hautement qualifiées dans le développement global de l'économie du savoir. Nous posons la question de savoir dans quelle mesure les mécanismes d'inclusion différentielle déterminent des flux de talents directionnels et non pas circulaires. Dès lors, l'injustice sociale globale est intimement liée à l'injustice cognitive globale: la migration hautement qualifiée se trouvant ainsi placée dans l'espace du mouvement néocolonial du capitalisme du savoir.

Mots-clés: division internationale du savoir; économie du savoir; justice cognitive; migrations; travailleurs qualifiés. 